\title{
Note on Prodi-Serrin-Ladyzhenskaya type regularity criteria for the Navier-Stokes equations
}

\author{
Chuong V. Tran, Xinwei Yu \\ Chuong V. Tran: School of Mathematics and Statistics, \\ University of St. Andrews, St Andrews KY16 9SS, United Kingdom* and \\ Xinwei Yu: Department of Mathematical and Statistical Sciences, \\ University of Alberta, Edmonton, AB, T6G 2G1, Canada ${ }^{\dagger}$ \\ (Dated: Jan. 1, 2016; Revised Oct. 23, 2016)
}

In this article we prove new regularity criteria of the Prodi-Serrin-Ladyzhenskaya type for the Cauchy problem of the three-dimensional incompressible Navier-Stokes equations. Our results improve the classical $L^{r}\left(0, T ; L^{s}\right)$ regularity criteria for both velocity and pressure by factors of certain negative powers of the scaling invariant norms $\|u\|_{L^{3}}$ and $\|u\|_{\dot{H}^{1 / 2}}$.

* cvt1@st-andrews.ac.uk

† xinwei2@ualberta.ca 


\section{INTRODUCTION}

The Cauchy problem of the three-dimensional incompressible Navier-Stokes equations play an important role in not only mathematical fluid mechanics but also the development of the theory of general evolutionary equations. The system reads

$$
\begin{aligned}
u_{t}+u \cdot \nabla u & =-\nabla p+\nu \triangle u, \quad(x, t) \in \mathbb{R}^{3} \times(0, \infty), \\
\operatorname{div} u & =0, \quad(x, t) \in \mathbb{R}^{3} \times(0, \infty), \\
u(x, 0) & =u_{0}(x), \quad x \in \mathbb{R}^{3},
\end{aligned}
$$

where $u: \mathbb{R}^{3} \mapsto \mathbb{R}^{3}$ is the velocity field, $p: \mathbb{R}^{3} \mapsto \mathbb{R}$ is the pressure, and $\nu>0$ is the (dimensionless) viscosity.

Systematic study of this problem began in 1934 with the classical paper [22] by Jean Leray, where it is shown that for arbitrary $T \in(0, \infty]$ there is at least one function $u(x, t)$ satisfying the following.

i. $u \in L^{\infty}\left(0, T ; L^{2}\left(\mathbb{R}^{d}\right)\right) \cap L^{2}\left(0, T ; H^{1}\left(\mathbb{R}^{d}\right)\right)$;

ii. $u$ satisfies (1) and (2) in the sense of distributions;

iii. $u$ takes the initial value in the $L^{2}$ sense: $\lim _{t \searrow 0}\left\|u(\cdot, t)-u_{0}(\cdot)\right\|_{L^{2}}=0$;

iv. $u$ satisfies the energy inequality

$$
\|u(\cdot, t)\|_{L^{2}}^{2}+2 \nu \int_{0}^{t}\|\nabla u(\cdot, \tau)\|_{L^{2}}^{2} \mathrm{~d} \tau \leqslant\left\|u_{0}\right\|_{L^{2}}^{2}
$$

for all $0 \leqslant t \leqslant T$.

Such a function $u(x, t)$ is called a Leray-Hopf weak solution for (1)-(3) in $\mathbb{R}^{d} \times[0, T)$.

It is easy to show that if a Leray-Hopf weak solution is smooth, then it is a classical solution and is furthermore unique (in the class of Leray-Hopf weak solutions). However the smoothness of Leray-Hopf weak solutions is still a completely open problem. On the other hand, it has been long known that various additional assumptions guarantee such smoothness. One important class of such assumptions is the following so-called Prodi-Serrin-Ladyzhenskaya criteria, developed over three decades in [11], [21], [24], [23], [25], [26]. If a Leray-Hopf solution $u(x, t)$ further satisfies

$$
u \in L^{r}\left(0, T ; L^{s}\left(\mathbb{R}^{3}\right)\right) \text { with } \frac{2}{r}+\frac{3}{s} \leqslant 1, \quad 3<s \leqslant \infty,
$$

then $u(x, t)$ is smooth. Here the norm of the mixed Lebesgue space $L^{r}\left(0, T: L^{s}\left(\mathbb{R}^{3}\right)\right)$ is defined as

$$
\|u\|_{L^{r}\left(0, T ; L^{s}\right)}:=\left\{\begin{array}{ll}
\left(\int_{0}^{T}\|u(\cdot, t)\|_{L^{s}}^{r}\right)^{1 / r} & 1 \leqslant r<\infty \\
\operatorname{esssup}_{t \in(0, T)}\|u(\cdot, t)\|_{L^{s}} & r=\infty
\end{array} .\right.
$$

The proof of the criterion (5) is quite straightforward through standard energy estimate, though it should be mentioned that it is the much more non-trivial "localized" version of (5) that was proved in the references above. The borderline case $u \in L^{\infty}\left(0, T ; L^{3}\left(\mathbb{R}^{3}\right)\right)$ that is missing in (5) turned out to be much more complicated due to the criticality of $\|u\|_{L^{3}}$ under the rescaling transformation $u(x, t) \mapsto \lambda u\left(\lambda x, \lambda^{2} t\right)$ that keeps (1)-(3) invariant. This case could not be dealt with using the method that established (5), and was only recently settled by Escauriaza, Seregin, and Šverák in 
[10] via a novel approach based on deep understanding of backward uniqueness of heat equations. Many generalizations and refinements of (5) have been proved, see e.g. [3], [5], [8], [12], [31], [34].

Mathematically the pressure $p$ serves as the Lagrange multiplier of the incompressibility constraint $\operatorname{div} u=0$. As a consequence there is no explicit equation governing the evolution of $p$ in (1)-(3). The lack of such an equation is partially compensated through the following relation between $u$ and $p$ obtained via taking divergence of (1),

$$
-\triangle p=\sum_{i, j=1}^{3} \partial_{i} \partial_{j}\left(u_{i} u_{j}\right) .
$$

For the convenience of analyzing (1)-(3) in the framework of functional analysis, (7) is usually written as

$$
p=\sum_{i, j=1}^{3} R_{i} R_{j}\left(u_{i} u_{j}\right),
$$

where $R_{i}, i=1,2,3$ are the Riesz transforms. As Riesz transforms are zeroth order pseudodifferential operators, there holds

$$
\|p\|_{L^{s}} \leqslant C\|u\|_{L^{2 s}}^{2} \text { for all } s \in(1, \infty) .
$$

From (9) it is natural to conjecture that

$$
p \in L^{r}\left(0, T ; L^{s}\right) \quad \text { with } \quad \frac{2}{r}+\frac{3}{s} \leqslant 2, \quad \frac{3}{2}<s \leqslant \infty
$$

may guarantee the smoothness of $u$. Note that thanks to (9), (10) is a weaker assumption than (5) as it is implied by the latter. The affirmative answer to this conjecture was established in [2], [7] and later refined in many follow-up papers, including [1], [3], [9], [13], [16], [17], [19], [27], [28], [30].

Roughly speaking, most of the aforementioned improvements of (5) or (10) can be categorized into two types. The first type replaces the Lebesgue norm $L^{s}$ and/or $L^{r}$ by weaker norms with the same scaling property. For example in [13] $\|p\|_{L^{s}}$ is replaced by the homogeneous Besov norm $\|p\|_{\dot{B}_{s, \sigma}^{0}}$ for some appropriate $\sigma$, and in [3] $\|u\|_{L^{s}}$ is replaced by $\|u\|_{L^{s, \infty}}$ where $L^{s, \infty}$ are the weak Lebesgue spaces. The second type of improvement weakens the conditions by a logarithmic factor. For example in [31] it is shown that $u$ is smooth as long as

$$
\int_{0}^{T} \frac{\|u\|_{L^{s}}^{r}}{\log \left(e+\|u\|_{L^{\infty}}\right)} \mathrm{d} t<\infty, \quad \frac{2}{r}+\frac{3}{s} \leqslant 1, \quad 3<s \leqslant \infty .
$$

Possibility of combining these two types of improvement has been well explored, see e.g. [12], [16]. We must also mention that criteria similar to (5) or (10) have also been proved for other physically meaningful quantities such as $\nabla u, \omega:=\operatorname{curl} u$, and $\nabla p$, see e.g. [4], [6], [14], [33], [35].

In this article we present and prove a new type of improvement of (5) and (10), of the form

$$
\int_{0}^{T} \frac{\|u\|_{L^{s}}^{r}}{\left(1+\|u\|_{X}\right)^{\kappa}} \mathrm{d} t<\infty, \quad \frac{2}{r}+\frac{3}{s} \leqslant 1, \quad 3<s<\infty
$$

or

$$
\int_{0}^{T} \frac{\|p\|_{L^{s}}^{r}}{\left(1+\|u\|_{X}\right)^{\kappa}} \mathrm{d} t<\infty, \quad \frac{2}{r}+\frac{3}{s} \leqslant 2, \quad \frac{3}{2}<s \leqslant \infty
$$


where $\|\cdot\|_{X}$ is a scaling-invariant norm for (1)-(3) and $\kappa>0$. More specifically, we will prove the following theorems.

Theorem 1. Let $u_{0} \in L^{2}\left(\mathbb{R}^{3}\right) \cap L^{q}\left(\mathbb{R}^{3}\right)$ for some $q>3$, and satisfy $\operatorname{div} u_{0}=0$. Let $u(t, x)$ be $a$ Leray-Hopf solution of NSE in $[0, T)$. If for some $s \in(3, \infty)$ and $\frac{2}{r}+\frac{3}{s}=1$ there holds

$$
\int_{0}^{T} \frac{\|u\|_{L^{s}}^{r}}{\left(1+\|u\|_{\dot{H}^{1 / 2}}\right)^{\kappa}} \mathrm{d} t<\infty
$$

where $\kappa=\left\{\begin{array}{ll}2 & 3<s \leqslant 5 \\ \frac{4}{s-3} & 5<s<\infty\end{array}\right.$, then $u(t, x)$ is smooth up to $T$ and could be extended beyond $T$.

Theorem 2. Let $u_{0} \in L^{2}\left(\mathbb{R}^{3}\right) \cap L^{q}\left(\mathbb{R}^{3}\right)$ for some $q>3$, and satisfy $\operatorname{div} u_{0}=0$. Let $u(t, x)$ be a Leray-Hopf solution of NSE in $[0, T)$. If for some $s \in(3, \infty)$ and $\frac{2}{r}+\frac{3}{s}=1$ there holds

$$
\int_{0}^{T} \frac{\|u\|_{L^{s}}^{r}}{\left(1+\|u\|_{L^{3}}\right)^{\kappa}} \mathrm{d} t<\infty
$$

where $\kappa=\left\{\begin{array}{ll}3 & 3<s \leqslant 5 \\ \frac{6}{s-3} & 5<s<\infty\end{array}\right.$, then $u(t, x)$ is smooth up to $T$ and could be extended beyond $T$.

Theorem 3. Let $u_{0} \in L^{2}\left(\mathbb{R}^{3}\right) \cap L^{q}\left(\mathbb{R}^{3}\right)$ for some $q>3$, and satisfy $\operatorname{div} u_{0}=0$. Let $u(t, x)$ be a Leray-Hopf solution of NSE in $[0, T)$. If for some $s \in\left(\frac{3}{2}, \infty\right)$ and $\frac{2}{r}+\frac{3}{s}=2$ there holds

$$
\int_{0}^{T} \frac{\|p\|_{L^{s}}^{r}}{\left(1+\|u\|_{\left.L^{3}\right)^{\kappa}}\right.} \mathrm{d} t<\infty
$$

where $\kappa=\left\{\begin{array}{ll}\frac{6}{2 s-3} & s \geqslant 3 \\ \frac{2 s}{2 s-3} & \frac{9}{4} \leqslant s \leqslant 3 \\ 3 & \frac{3}{2}<s \leqslant \frac{9}{4}\end{array}\right.$, then $u(t, x)$ is smooth up to $T$ and could be extended beyond $T$.

A few remarks are in order.

Remark 1. It is straightforward to cover the sub-critical cases. For example, if u satisfies (14) for some $r, s$ satisfying $s \in(3, \infty)$ and $\frac{2}{r}+\frac{3}{s}<1$, then there is $r^{\prime}<r$ such that $\frac{2}{r^{\prime}}+\frac{3}{s}=1$ and (14) holds for $r^{\prime}, s$ thanks to Hölder's inequality.

Remark 2. We emphasize that unlike (5) and (10), where one is weaker than the other, Theorems 1, 2, and 3 are independent of each other. For example, although $\|u\|_{L^{3}} \leqslant C\|u\|_{\dot{H}^{1 / 2}}$ by Sobolev embedding, Theorem 2 does not follow from Theorems 1, as the values of $\kappa$ are different.

Remark 3. When $s=6$, criterion (15) can be seen to be comparable but not equivalent to the following criterion

$$
\int_{0}^{T} \frac{\|u\|_{L^{6}}^{6}}{\|u\|_{L^{4}}^{4}+2\|u\|_{\dot{H}^{1}}^{2}}<\infty
$$

which has been reported recently [29]. The two criteria would be equivalent if we could establish that $\|u\|_{L^{4}}^{4}+2\|u\|_{\dot{H}^{1}}^{2} \approx\left(\|u\|_{L^{3}}^{2}+2\right)\|u\|_{L^{6}}^{2}$, which is consistent in scaling yet does not hold for general divergence free vector field $u$. For all other $s>3$, (15) may be regarded as an extention of (17). It is also of interest to explore the possibility of weakening (16) through replacing the pressure $p$ by the "effective pressure" $p+\mathcal{P}$ in [29]. 
Remark 4. It is not clear whether the splitting into different cases in Theorems 1, 2, and 3 is purely technical, due to the limitations of the energy method, or reflects deeper properties of the Navier-Stokes dynamics.

Remark 5. From the proofs we will see that a logarithmic factor could easily be added "for free". For example, (15) could be replaced by

$$
\int_{0}^{T} \frac{\|u\|_{L^{s}}^{r}}{\left(1+\|u\|_{L^{3}}\right)^{\kappa} \log \left(e+\|u\|_{L^{3}}\right)} \mathrm{d} t<\infty
$$

However it does not seem likely that the $\|u\|_{L^{3}}$ inside the logarithm could be replaced by $\|u\|_{L^{\infty}}$. Thus our results are not stronger than, though still independent of, the previous logarithmic improvement results such as (11).

Remark 6. Criteria in a sense similar to Theorems 1, 2, and 3 have been proved in [18] and [32]. There it is shown that smoothness of the solution u is guaranteed if one of the following holds.

- [32].

$$
\frac{p}{1+|u|^{\delta}} \in L^{r}\left(0, T ; L^{s}\right), \quad \frac{2}{r}+\frac{3}{s}=\frac{5-3 \delta}{2}, \quad \frac{6}{5-3 \delta}<s \leqslant \infty, \quad 1 \leqslant \delta \leqslant \frac{5}{3} ;
$$

- $[18]$.

$$
\frac{p}{1+|u|^{\delta}} \in L^{r}\left(0, T ; L^{s}\right), \quad \text { with } \frac{2}{r}+\frac{3}{s}=\frac{4-3 \delta}{2}, \quad \frac{18}{8-9 \delta} \leqslant s \leqslant \frac{6}{2-3 \delta}, \quad 0 \leqslant \delta<\frac{2}{3} .
$$

- [18].

$$
\frac{p}{1+|u|^{\delta}} \in L^{r}\left(0, T ; L^{s}\right), \quad \text { with } \frac{2}{r}+\frac{3}{s}=\frac{4-3 \delta}{2}, \quad \frac{18}{8-9 \delta} \leqslant s \leqslant \frac{6}{2-3 \delta}, \quad \frac{2}{3} \leqslant \delta \leqslant \frac{8}{9} .
$$

We briefly discuss their relations to Theorems 1, 2, and 3.

1. (19)-(21) are sub-critical from a scaling point of view and therefore does not improve (10) except for the case $\delta=1$ in (19) (and $\delta=0$ in (20) which reduces (20) to (10)). To see this we recall (8) which dictates that $p$ scales as $|u|^{2}$. This makes $\frac{p}{1+|u|^{\delta}} \in L^{r}\left(0, T ; L^{s}\right)$ roughly equivalent to, from the scaling point of view, $|u|^{2-\delta} \in L^{r}\left(0, T ; L^{s}\right)$ that is $u \in L^{(2-\delta) r}\left(0, T ; L^{(2-\delta) s}\right)$. Thus for example (20) corresponds to

$$
\frac{2}{(2-\delta) r}+\frac{3}{(2-\delta) s}=\frac{4-3 \delta}{4-2 \delta}<1
$$

for all values of $\delta$ except $\delta=0$. Similarly, in (19) we have

$$
\frac{2}{(2-\delta) r}+\frac{3}{(2-\delta) s}=1+\frac{1-\delta}{4-2 \delta}<1
$$

unless $\delta=1$. 
2. On the other hand, in (19)-(21) the factor $\left(1+|u|^{\delta}\right)^{-1}$ is inside the whole space-time integral, while in our theorems $\left(1+\|u\|_{L^{3}}\right)^{-\kappa}$ or $\left(1+\|u\|_{\dot{H}^{1 / 2}}\right)^{-\kappa}$ is only inside the time integral. Thus the conditions (19)-(21) are in a sense more "localized".

Remark 7. It is quite straightforward to generalize Theorems 1, 2, and 3 to d-dimensional NavierStokes equations. For simplicity of presentation we will focus on the physical case $d=3$ in this article.

In the next section we prove Theorems 1,2 , and 3.

\section{PROOF OF THEOREMS}

\section{A. Preliminaries}

Without loss of generality, we take $\nu=1$ in (1) to simplify the presentation. We apply the following result, summarized from [15], [20], to guarantee short-time smoothness of the solution and thus relieving us from worrying about the legitimacy of the various integral and differential manipulations below.

Theorem 4. Let $u_{0} \in L^{s}\left(\mathbb{R}^{3}\right), s \geqslant 3$. Then there exists $T>0$ and a unique classical solution $u \in \mathrm{BC}\left(0, T ; L^{s}\left(\mathbb{R}^{3}\right)\right)$. Moreover, let $\left(0, T_{*}\right)$ be the maximal interval such that the solution u stays in $C\left(0, T_{*} ; L^{s}\left(\mathbb{R}^{3}\right)\right), s>3$. Then for any $t \in\left(0, T_{*}\right)$,

$$
\|u(\cdot, t)\|_{L^{s}} \geqslant \frac{C}{\left(T_{*}-t\right)^{\frac{s-3}{2 s}}}
$$

where the constant $C$ is independent of $T_{*}$ and $s$.

We will also need the following simple lemma.

Lemma 1. Let $X(t) \in C^{1}(0, T) \cap C([0, T))$ be non-negative and solve $\dot{X}(t) \leqslant \frac{A(t)}{X(t)^{\kappa}} X(t)+\frac{C}{X(t)^{\kappa}}$ for some $A(t) \geqslant 0, k>0$. Assume

$$
\int_{0}^{T} \frac{A(t)}{(1+X(t))^{\kappa}} \mathrm{d} t<\infty
$$

Then $\lim \sup _{t \rightarrow T-} X(t)<\infty$.

Proof. Denote $B(t):=\frac{A(t)}{\max \{1, X(t)\}^{\kappa}}$. It is clear that $(25)$ is equivalent to $\int_{0}^{T} B(t) \mathrm{d} t<\infty$. Let $Y(t):=\max \{1, X(t)\}$. Then $Y(t)=X(t)$ on the union of at most countably many open intervals $\left(t_{i L}, t_{i R}\right)$ with $Y\left(t_{i L}\right)=1$. Now on $\left(t_{i L}, t_{i R}\right)$ we have $Y(t)>1$ and therefore

$$
\begin{aligned}
\dot{Y}(t) & \leqslant \frac{A(t)}{X(t)^{k}} X(t)+\frac{C}{X(t)^{k}} \\
& =\frac{A(t)}{Y(t)^{k}} Y(t)+\frac{C}{Y(t)^{k}} \\
& \leqslant B(t) Y(t)+C .
\end{aligned}
$$

The conclusion immediately follows. 
Finally we need the following result which is a special case of Theorem 1.3 in [10].

Theorem 5. Suppose that $u$ is a weak Leray-Hopf solution of the Cauchy problem (1)-(3). If furthermore $u \in L^{\infty}\left(0, T ; L^{3}\left(\mathbb{R}^{3}\right)\right)$, then $u$ is smooth up to $T$ and can be extended beyond $T$.

Notation. In the following we will use $A \lesssim B$ to denote $A \leqslant c B$ when the constant $c$ is inconsequential to the validity of the proof.

\section{B. Proof of Theorem 1}

Assume the contrary. By Theorem 4 there is $T^{*} \in(0, T)$ such that $u(x, t)$ is smooth for $t \in\left(0, T^{*}\right)$ but cease to be so at $t=T^{*}$. Thanks to Theorem 5 and the Sobolev embedding $\dot{H}^{1 / 2}\left(\mathbb{R}^{3}\right) \hookrightarrow$ $L^{3}\left(\mathbb{R}^{3}\right)$, this implies $\lim \sup _{t} T_{T^{*}}\|u\|_{\dot{H}^{1 / 2}}=\infty$. Therefore to obtain contradiction it suffices to prove that $\|u\|_{\dot{H}^{1 / 2}} \leqslant C, \forall t \in\left(0, T^{*}\right)$, for some constant $C>0$.

Let $\Lambda:=(-\triangle)^{1 / 2}$. Multiplying the equation by $\Lambda u$ and then integrate over $\mathbb{R}^{3}$, we reach

$$
\begin{aligned}
\frac{1}{2} \frac{\mathrm{d}}{\mathrm{d} t}\|u\|_{\dot{H}^{1 / 2}}^{2} & =-\int_{\mathbb{R}^{3}} u \cdot \nabla u \cdot \Lambda u \mathrm{~d} x-\int_{\mathbb{R}^{3}} \Lambda u \cdot \Lambda^{2} u \mathrm{~d} x \\
& \leqslant \int_{\mathbb{R}^{3}}|u|\left|\nabla u\|\Lambda u \mid \mathrm{d} x-\| u \|_{\dot{H}^{3 / 2}}^{2} .\right.
\end{aligned}
$$

In what follows we discuss the two regimes of $s$ stated in the theorem separately.

- $3<s \leqslant 5$.

Let $s^{\prime}$ be the conjugate to $s$, that is $\frac{1}{s}+\frac{1}{s^{\prime}}=1$. We start by estimating using Hölder's inequality

$$
I:=\int_{\mathbb{R}^{3}}|u|\left|\nabla u\|\Lambda u \mid \mathrm{d} x \leqslant\| u\left\|_{L^{s}}\right\| \nabla u\left\|_{L^{2 s^{\prime}}}\right\| \Lambda u \|_{L^{2 s^{\prime}}}\right.
$$

Next we notice that as $3<s \leqslant 5 \Longrightarrow 2 s^{\prime} \in\left[\frac{5}{2}, 3\right) \subset(1, \infty)$, the boundedness of Riesz transforms on $L^{p}\left(\mathbb{R}^{3}\right)$ for $1<p<\infty$ now yields $\|\Lambda u\|_{L^{2 s^{\prime}}} \lesssim\|\nabla u\|_{L^{2 s^{\prime}}}$ since $\Lambda u=-\Lambda^{-1} \nabla$. $(\nabla u)=-\sum_{i=1}^{3} R_{i}\left(\partial_{i} u\right)$. Consequently

$$
I \lesssim\|u\|_{L^{s}}\|\nabla u\|_{L^{2 s^{\prime}}}^{2}
$$

Now thanks to the the interpolation inequality

$$
\|\nabla u\|_{L^{2 s^{\prime}}} \lesssim\|u\|_{L^{s}}^{\frac{s-3}{6}}\|u\|_{\dot{H}^{3 / 2}}^{\frac{9-s}{6}}
$$

we further obtain

$$
I \lesssim\|u\|_{L^{s}}\|u\|_{L^{s}}^{(s-3) / 3}\|u\|_{\dot{H}^{3 / 2}}^{(9-s) / 3} .
$$

Finally by Young's inequality we conclude that

$$
I \leqslant C\|u\|_{L^{s}}^{r}+\frac{1}{2}\|u\|_{\dot{H}^{3 / 2}}^{2}
$$


with $r=\frac{2 s}{s-3}$.

Substituting (32) into (27), we have

$$
\|u\|_{\dot{H}^{1 / 2}} \frac{\mathrm{d}}{\mathrm{d} t}\|u\|_{\dot{H}^{1 / 2}} \lesssim\|u\|_{L^{s}}^{r}
$$

which gives

$$
\frac{\mathrm{d}}{\mathrm{d} t}\|u\|_{\dot{H}^{1 / 2}} \lesssim \frac{\|u\|_{L^{s}}^{r}}{\|u\|_{\dot{H}^{1 / 2}}^{2}}\|u\|_{\dot{H}^{1 / 2}}
$$

and the conclusion follows from Lemma 1.

- $5<s<\infty$.

We first notice that

$$
\int_{\mathbb{R}^{3}}|u||\nabla u||\Lambda u| \mathrm{d} x \leqslant \int_{\mathbb{R}^{3}}|u||\nabla u|^{2} \mathrm{~d} x+\int_{\mathbb{R}^{3}}|u||\Lambda u|^{2} \mathrm{~d} x=: I+I I .
$$

We estimate $I$ first. By Hölder inequality we have

$$
\begin{aligned}
I & =\int_{\mathbb{R}^{3}}|u \| \nabla u|^{\frac{2(s-5)}{s-2}}|\nabla u|^{\frac{6}{s-2}} \mathrm{~d} x \\
& \leqslant\|u\|_{L^{s}}\left\||\nabla u|^{\frac{2(s-5)}{s-2}}\right\|_{L^{\frac{s-2}{s-5}}}\left\||\nabla u|^{\frac{6}{s-2}}\right\|_{L^{\frac{s(s-2)}{2(s+1)}}} \\
& =\|u\|_{L^{s}}\|\| \nabla u\left\|_{L^{2-2}}^{\frac{2(s-5)}{s-2}}\right\| \nabla u \|_{L^{\frac{3 s}{s+1}}}^{\frac{6}{s-2}} .
\end{aligned}
$$

Note that as $s>5$, there holds $1<\frac{s-2}{s-5}<\infty$ and $1<\frac{s}{4}<\frac{s(s-2)}{2(s+1)}<\infty$. Therefore the application of Hölder inequality is justified.

Next we apply the following Gagliardo-Nirenberg inequalities,

$$
\|\nabla u\|_{L^{2}} \lesssim\|u\|_{\dot{H}^{1 / 2}}^{1 / 2}\|u\|_{\dot{H}^{3 / 2}}^{1 / 2}, \quad\|\nabla u\|_{L^{3 s /(s+1)}} \lesssim\|u\|_{L^{s}}^{1 / 3}\|u\|_{\dot{H}^{3 / 2}}^{2 / 3},
$$

to obtain

$$
I \lesssim\|u\|_{L^{s}}^{s /(s-2)}\|u\|_{\dot{H}^{1 / 2}}^{(s-5) /(s-2)}\|u\|_{\dot{H}^{3 / 2}}^{(s-1) /(s-2)} .
$$

Young's inequality now yields

$$
I \leqslant C\|u\|_{L^{s}}^{2 s /(s-3)}\|u\|_{\dot{H}^{1 / 2}}^{2(s-5) /(s-3)}+\frac{1}{2}\|u\|_{\dot{H}^{3 / 2}}^{2} .
$$

Through almost identical arguments, the same estimate could be obtained for $I I$.

$$
I I \leqslant C\|u\|_{L^{s}}^{2 s /(s-3)}\|u\|_{\dot{H}^{1 / 2}}^{2(s-5) /(s-3)}+\frac{1}{2}\|u\|_{\dot{H}^{3 / 2}}^{2} .
$$

Substituting these into (27) and dividing both sides by $\|u\|_{\dot{H}^{1 / 2}}$, we obtain

$$
\frac{\mathrm{d}}{\mathrm{d} t}\|u\|_{\dot{H}^{1 / 2}} \lesssim \frac{\|u\|_{L^{s}}^{r}}{\|u\|_{\dot{H}^{1 / 2}}^{4 /(s-3)}}\|u\|_{\dot{H}^{1 / 2}}
$$

and the conclusion now follows from Lemma 1. 


\section{Proof of Theorem 2}

Similar to the proof of Theorem 1, we assume that $u$ blows up at $T^{*} \in(0, T)$, and it suffices to show that $\|u\|_{L^{3}} \leqslant C$ on $\left(0, T^{*}\right)$ for some $C>0$ independent of $t$.

We multiply (1) by $|u| u$. and integrate in $\mathbb{R}^{3}$ to obtain

$$
\begin{aligned}
\|u\|_{L^{3}}^{2} \frac{\mathrm{d}}{\mathrm{d} t}\|u\|_{L^{3}} & =-\int_{\mathbb{R}^{3}}|u| u \cdot \nabla p \mathrm{~d} x+\int_{\mathbb{R}^{3}}|u| u \cdot \Delta u \mathrm{~d} x \\
& =\int_{\mathbb{R}^{3}} p u \cdot \nabla(|u|) \mathrm{d} x+\int_{\mathbb{R}^{3}}|u| u \cdot \Delta u \mathrm{~d} x \\
& =\int_{\mathbb{R}^{3}} p|u|(\hat{u} \cdot \nabla|u|) \mathrm{d} x+\int_{\mathbb{R}^{3}}|u| u \cdot \triangle u \mathrm{~d} x .
\end{aligned}
$$

where $\hat{u}:=\frac{u}{|u|}$ (if $u=0$, just defined $\hat{u}=0$ too).

Recalling the identity

$$
u \cdot \triangle u=\nabla \cdot(|u| \nabla|u|)-|\nabla u|^{2}
$$

we easily derive

$$
\int_{\mathbb{R}^{3}}|u| u \cdot \triangle u \mathrm{~d} x=-\frac{4}{9}\left\|\nabla|u|^{3 / 2}\right\|_{L^{2}}^{2}-\left\|\left|\nabla u\left\|\left.u\right|^{1 / 2}\right\|_{L^{2}}^{2},\right.\right.
$$

and reach the following estimate

$$
\frac{\mathrm{d}}{\mathrm{d} t}\|u\|_{L^{3}}^{3}+\left\||\nabla u||u|^{1 / 2}\right\|_{L^{2}}^{2}+\left\|\nabla|u|^{3 / 2}\right\|_{L^{2}}^{2} \lesssim\left|\int_{\mathbb{R}^{3}} p\right| u|(\hat{u} \cdot \nabla|u|) \mathrm{d} x| .
$$

which gives

$$
\|u\|_{L^{3}}^{2} \frac{\mathrm{d}}{\mathrm{d} t}\|u\|_{L^{3}} \lesssim C \int_{\mathbb{R}^{3}}\left|p \left\|u | ^ { \frac { 1 } { 2 } } | \nabla | u | ^ { 3 / 2 } \left|\mathrm{~d} x-\left\||u|^{3 / 2}\right\|_{\dot{H}^{1}}^{2} .\right.\right.\right.
$$

Application of Young's inequality and then Hölder and Sobolev inequalities to (46) gives

$$
\begin{aligned}
\|u\|_{L^{3}}^{2} \frac{\mathrm{d}}{\mathrm{d} t}\|u\|_{L^{3}} & \lesssim C \int_{\mathbb{R}^{3}}|p|^{2}|u| \mathrm{d} x-\left\||u|^{3 / 2}\right\|_{\dot{H}^{1}}^{2} \\
& \lesssim C\left\|p^{2}\right\|_{L^{5 / 4}}\|u\|_{L^{5}}-\|u\|_{L^{9}}^{3} \\
& =C\|p\|_{L^{5 / 2}}^{2}\|u\|_{L^{5}}-\|u\|_{L^{9}}^{3} \\
& \lesssim C\|u\|_{L^{5}}^{5}-\|u\|_{L^{9}}^{3} .
\end{aligned}
$$

Note that in the last inequality we have used (9).

In what follows we discuss the two regimes of $s$ stated in the theorem separately.

- $3<s \leqslant 5$. In this case we apply the interpolation inequality

$$
\|u\|_{L^{5}} \leqslant\|u\|_{L^{s}}^{\theta}\|u\|_{L^{9}}^{1-\theta}
$$

where $\theta=\frac{4}{5} \frac{s}{9-s}$. This gives

$$
\begin{aligned}
\|u\|_{L^{3}}^{2} \frac{\mathrm{d}}{\mathrm{d} t}\|u\|_{L^{3}} & \lesssim C\|u\|_{L^{s}}^{4 s /(9-s)}\|u\|_{L^{9}}^{9(5-s) /(9-s)}-\|u\|_{L^{9}}^{3} \\
& \lesssim C\|u\|_{L^{s}}^{2 s /(s-3)}
\end{aligned}
$$


after application of Young's inequality. Dividing both sides by $\|u\|_{L^{3}}^{2}$ we have

$$
\frac{\mathrm{d}}{\mathrm{d} t}\|u\|_{L^{3}} \lesssim \frac{\|u\|_{L^{s}}^{2 s /(s-3)}}{\|u\|_{L^{3}}^{3}}\|u\|_{L^{3}}
$$

The conclusion now follows from Lemma 1.

- $5<s<\infty$. In this case we apply the interpolation inequality

$$
\|u\|_{L^{5}} \leqslant\|u\|_{L^{3}}^{\theta}\|u\|_{L^{s}}^{1-\theta}
$$

where $\theta=\frac{3}{5} \frac{s-5}{s-3}$. This gives

$$
\|u\|_{L^{3}}^{2} \frac{\mathrm{d}}{\mathrm{d} t}\|u\|_{L^{3}} \lesssim\|u\|_{L^{3}}^{3(s-5) /(s-3)}\|u\|_{L^{s}}^{2 s /(s-3)} .
$$

Dividing both sides by $\|u\|_{L^{3}}^{2}$ we have

$$
\frac{\mathrm{d}}{\mathrm{d} t}\|u\|_{L^{3}} \lesssim \frac{\|u\|_{L^{s}}^{2 s /(s-3)}}{\|u\|_{L^{3}}^{6 /(s-3)}}\|u\|_{L^{3}},
$$

and the conclusion immediately follows from Lemma 1.

\section{Proof of Theorem 3}

Again we assume that $u$ blows up at $T^{*} \in(0, T)$, and try to to show that $\|u\|_{L^{3}} \leqslant C$ on $\left(0, T^{*}\right)$ for some $C>0$ independent of $t$.

Following (42)-(45) we have

$$
\begin{aligned}
\|u\|_{L^{3}}^{2} \frac{\mathrm{d}}{\mathrm{d} t}\|u\|_{L^{3}} & \leqslant \int_{\mathbb{R}^{3}}|p\|u\| \nabla| u\|\mathrm{~d} x-\| \nabla|u|^{3 / 2} \|_{L^{2}}^{2} \\
& =\left.\int_{\mathbb{R}^{3}}|p|^{\alpha}|p|^{1-\alpha}|u|^{1 / 2}|\nabla| u\right|^{3 / 2}\left|\mathrm{~d} x-\left\|\nabla|u|^{3 / 2}\right\|_{L^{2}}^{2}\right. \\
& \leqslant\left\|||^{\alpha}\right\|_{L^{a}}\left\||p|^{1-\alpha}\right\|_{L^{b}}\left\||u|^{1 / 2}\right\|_{L^{c}}\left\|\nabla|u|^{3 / 2}\right\|_{L^{2}}-\left\|\nabla|u|^{3 / 2}\right\|_{L^{2}}^{2} \\
& =\|p\|_{L^{a \alpha}}^{\alpha}\|p\|_{L^{b(1-\alpha)}}^{1-\alpha}\|u\|_{L^{c / 2}}^{1 / 2}\left\|\nabla|u|^{3 / 2}\right\|_{L^{2}}-\left\|\nabla|u|^{3 / 2}\right\|_{L^{2}}^{2} \\
& \leqslant C\|p\|_{L^{s}}^{\alpha}\|u\|_{L^{2 b(1-\alpha)}}^{2(1-\alpha)}\|u\|_{L^{c / 2}}^{1 / 2}\left\|\nabla|u|^{3 / 2}\right\|_{L^{2}}-\left\|\nabla|u|^{3 / 2}\right\|_{L^{2}}^{2} \\
& =: A-\left\|\nabla|u|^{3 / 2}\right\|_{L^{2}}^{2} .
\end{aligned}
$$

for appropriate $\alpha, a, b, c$ with $a \alpha=s, \frac{1}{a}+\frac{1}{b}+\frac{1}{c}=\frac{1}{2}$. We deal with the three cases stated in the theorem one by one.

- $3<s<\infty$. We take $\alpha=\frac{s}{2 s-3}, a=2 s-3, b=\frac{3(2 s-3)}{2 s-6}, c=6$. This gives

$$
A=C\|p\|_{L^{s}}^{\frac{s}{2 s-3}}\|u\|_{L^{3}}^{\frac{6 s-15}{4 s-6}}\left\|\nabla|u|^{3 / 2}\right\|_{L^{2}} \leqslant C\|p\|_{L^{s}}^{\frac{2 s}{2 s-3}}\|u\|_{L^{3}}^{\frac{6 s-15}{2 s-3}}+\left\|\nabla|u|^{3 / 2}\right\|_{L^{2}}^{2} .
$$


Here we have applied Young's inequality. Substituting (55) into (54) and dividing both sides by $\|u\|_{L^{3}}^{2}$, we reach

$$
\frac{\mathrm{d}}{\mathrm{d} t}\|u\|_{L^{3}} \leqslant \frac{\|p\|_{L^{s}}^{\frac{2 s}{2 s-3}}}{\|u\|_{L^{3}}^{\frac{6}{2 s-3}}}\|u\|_{L^{3}} .
$$

The conclusion now follows from Lemma 1.

- $\frac{9}{4}<s<3$. We take $\alpha=1, a=s, c=\frac{2 s}{s-2}$. Note that in this case the term involving $b$ is not present. Application of the following interpolation inequality and Sobolev inequality

$$
\|u\|_{L^{\frac{s}{s-2}}} \leqslant\|u\|_{L^{3}}^{\frac{4 s-9}{s}}\|u\|_{L^{9}}^{\frac{9-3 s}{s}}, \quad\|u\|_{L^{9}}^{3} \lesssim\left\|\nabla|u|^{3 / 2}\right\|_{L^{2}}^{2}
$$

gives

$$
A=C\|p\|_{L^{s}}\|u\|_{L^{s /(s-2)}}^{1 / 2}\left\|\nabla|u|^{3 / 2}\right\|_{L^{2}} \lesssim\|p\|_{L^{s}}\|u\|_{L^{3}}^{\frac{4 s-9}{2 s}}\left\|\nabla|u|^{3 / 2}\right\|_{L^{2}}^{\frac{3}{s}} .
$$

Application of Young's inequality now gives

$$
A \leqslant C\|p\|_{L^{s}}^{\frac{2 s}{2 s-3}}\|u\|_{L^{3}}^{\frac{4 s-9}{2 s-3}}+\left\|\nabla|u|^{3 / 2}\right\|_{L^{2}}^{2} .
$$

Substituting this into (54) and dividing both sides by $\|u\|_{L^{3}}^{2}$, we reach

$$
\frac{\mathrm{d}}{\mathrm{d} t}\|u\|_{L^{3}} \leqslant \frac{\|p\|_{L^{s}}^{\frac{2 s}{2 s-3}}}{\|u\|_{L^{3}}^{\frac{2 s}{2 s-3}}}\|u\|_{L^{3}} .
$$

The conclusion now follows from Lemma 1.

- $\frac{3}{2}<s \leqslant \frac{9}{4}$. We take $\alpha=\frac{2 s}{9-2 s}, a=\frac{9-2 s}{2}, b=\frac{9}{2} \frac{9-2 s}{9-4 s}, c=18$. Now application of Sobolev inequality $\|u\|_{L^{9}}^{3} \lesssim\left\|\nabla|u|^{3 / 2}\right\|_{L^{2}}^{2}$ gives

$$
A=C\|p\|_{L^{s}}^{\frac{2 s}{9-2 s}}\|u\|_{L^{9}}^{\frac{45-18 s}{18-4 s}}\left\|\nabla|u|^{3 / 2}\right\|_{L^{2}} \lesssim\|p\|_{L^{s}}^{\frac{2 s}{9-2 s}}\left\|\nabla|u|^{3 / 2}\right\|_{L^{2}}^{\frac{24-8 s}{9-2 s}}
$$

Note that since $\frac{3}{2}<s \leqslant \frac{9}{4}$, there holds $\frac{24-8 s}{9-2 s}<2$. Thus we can apply Young's inequality and obtain

$$
A \leqslant C\|p\|_{L^{s}}^{\frac{2 s}{2 s-3}}+\left\|\nabla|u|^{3 / 2}\right\|_{L^{2}}^{2}
$$

Substituting this into (54) and dividing both sides by $\|u\|_{L^{3}}^{2}$, we reach

$$
\frac{\mathrm{d}}{\mathrm{d} t}\|u\|_{L^{3}} \leqslant \frac{\|p\|_{L^{s}}^{\frac{2 s}{2 s-3}}}{\|u\|_{L^{3}}^{3}}\|u\|_{L^{3}} .
$$

The conclusion now follows from Lemma 1. 
Acknowledgment. Part of this research was carried out when CVT was visiting the University of Alberta, whose hospitality is gratefully acknowledged. XY is partially supported by the Discovery Grant RES0020476 from NSERC. The authors would like to thank the associate editor and the anonymous referee for the valuable comments and suggestions. The authors would also like to thank Prof. Edriss Titi for helpful discussions.

[1] Samir Benbernou. A note on the regularity criterion in terms of pressure for the Navier-Stokes equations. Applied Mathematics Letters, 22(9):1438-1443, September 2009.

[2] Luigi C. Berselli and Giovanni P. Galdi. Regularity criteria involving the pressure for the weak solutions to the Navier-Stokes equations. Proc. Amer. Math. Soc., 130:3585-3595, 2002.

[3] Stefano Bosia, Vittorino Pata and James C. Robinson. A weak- $L^{p}$ Prodi-Serrin type regularity criterion for the Navier-Stokes equations. J. Math. Fluid Mech., 16(4): 721-725, 2014.

[4] Chongsheng Cao, Junlin Qin, and Edriss S. Titi. Regularity criterion for solutions of three-dimensional turbulent channel flows. Commun. PDE, 33:419-428, 2008.

[5] Chongsheng Cao and Edriss S. Titi. Regularity criteria for the three-dimensional Navier-Stokes equations. Indiana University Mathematics Journal, 57 (6): 2643-2661, 2008.

[6] Chongsheng Cao and Edriss S. Titi. Global regularity criterion for the 3D Navier-Stokes equations involving one entry of the velocity gradient tensor. Arch. Rational Mech. Anal., 202: 919-932, 2011.

[7] Dongho Chae and Jihoon Lee. Regularity criterion in terms of pressure for the Navier-Stokes equations. Nonlinear Analysis: Theory, Methods \&6 Applications, 46(5):727-735, November 2001.

[8] Chi Hin Chan and Alexis Vasseur. Log improvement of the Prodi-Serrin criteria for Navier-Stokes equations. Methods and Applications of Analysis, 14(2):197-212, 2007.

[9] Qionglei Chen and Zhifei Zhang. Regularity criterion via the pressure on weak solutions to the 3D Navier-Stokes equations. Proc. Amer. Math. Soc., 135:1829-1837, 2007.

[10] L. Escauriaza, G. Seregin and V. Šverák. $L_{3, \infty}$-solutions of Navier-Stokes equations and backward uniqueness. Uspekhi Mat. Nauk, 58(2):3-44, 2003. Russian Math. Surveys 58(2): 211-250.

[11] E. B. Fabes, B. F. Jones and N. M. Riviere. The initial value problem for the Navier-Stokes equations with data in $L^{p}$. Arch. Rational Mech. Anal., 45:222-248, 1972.

[12] Jishan Fan, Song Jiang, Gen Nakamura and Yong Zhou. Logarithmically improved regularity criteria for the Navier-Stokes and MHD equations. Journal of Mathematical Fluid Mechanics, 13(4):557-571, December 2011.

[13] Jishan Fan, Song Jiang and Guoxi Ni. On regularity criteria for the n-dimensional Navier-Stokes equations in terms of the pressure. Journal of Differential Equations, 244(11):2963-2979, June 2008.

[14] Sadek Gala. Remarks on regularity criterion for weak solutions to the Navier-Stokes equations in terms of the gradient of the pressure. Applicable Analysis: An International Journal, 92(1):96-103, 2013.

[15] Yoshikazu Giga. Solutions for semilinear parabolic equations in $L^{p}$ and regularity of weak solutions of the Navier-Stokes system. Journal of Differential Equations, 62(2):186-212, April 1986.

[16] Zhengguang Guo and Sadek Gala. Remarks on logarithmical regularity criteria for the Navier-Stokes equations. Journal of Mathematical Physics, 52(6):63503, 2011.

[17] Zhengguang Guo and Sadek Gala. A note on the regularity criteria for the Navier-Stokes equations. Applied Mathematics Letters, 25(3):305-309, March 2012.

[18] Zhengguang Guo, Peter Wittwer and Weiming Wang. Regularity issue of the Navier-Stokes equations involving the combination of pressure and velocity field. Acta Applicandae Mathematicae, 123(1):99112, February 2013.

[19] Xiaowei He and Sadek Gala. Regularity criterion for weak solutions to the Navier-Stokes equations in terms of the pressure in the class $\left.L^{2}\left(0, T ; \dot{B}_{\infty, \infty}^{-1}\left(R^{3}\right)\right)\right\}$. Nonlinear Analysis: Real World Applications, 12(6):3602-3607, December 2011.

[20] Tosio Kato. Strong $L^{p}$-solutions of the Navier-Stokes equation in $R^{m}$, with applications to weak solutions. Math. Z., 187:471-480, 1984. 
[21] O. A. Ladyzhenskaya. On uniqueness and smoothness of generalized solutions to the Navier-Stokes equations. Zap. Nauchn. Sem. Leningrad. Otdel. Mat. Inst. Steklov. (LOMI), 5:169-185, 1967. English transl., Sem. Math. V. A. Steklov Math. Inst. Leningrad 5 (1969), 60-66.

[22] Jean Leray. On the motion of a viscous liquid filling space. Acta mathematica, 63:193-248, 1934.

[23] T. Ohyama. Interior regularity of weak solutions to the Navier-Stokes equation. Proc. Japan Acad., 36:273-277, 1960

[24] G. Prodi. Un teorema di unicità per le equazioni di Navier-Stokes. Ann. Mat. Pura Appl., 4(48):173$182,1959$.

[25] Jame Serrin. On the interior regularity of weak solutions of the Navier-Stokes equations. Arch. Ration. Mech. Anal., 9:187-191, 1962.

[26] Michael Struwe. On partial regularity results for the Navier-Stokes equations. Comm. Pure Appl. Math., 41(4):437-458, 1988.

[27] Michael Struwe. On a Serrin-type regularity criterion for the Navier-Stokes equations in terms of the pressure. Journal of Mathematical Fluid Mechanics, 9(2):235-242, June 2007.

[28] Tomoyuki Suzuki. A remark on the regularity of weak solutions to the Navier-Stokes equations in terms of the pressure in Lorentz spaces. Nonlinear Analysis: Theory, Methods 85 Applications, 75(9):38493853, June 2012

[29] C.V. Tran and X. Yu. Pressure moderation and effective pressure in Navier-Stokes flows. Nonlinearity, 29: 2990-3005, 2016

[30] Xingwei Zhang, Yan Jia and Bo-Qing Dong. On the pressure regularity criterion of the 3D Navier-Stokes equations. Journal of Mathematical Analysis and Applications, 393(2):413-420, September 2012.

[31] Yi Zhou and Zhen Lei. Logarithmically improved criteria for Euler and Navier-Stokes equations. Communications on Pure and Applied Analysis, 12(6):2715-2719, November 2013.

[32] Yong Zhou. Regularity criteria in terms of pressure for the 3-D Navier-Stokes equations in a generic domain. Mathematische Annalen, 328(1):173-192, January 2004

[33] Yong Zhou. On regularity criteria in terms of pressure for the Navier-Stokes equations in $R^{3}$. Proc. Amer. Math. Soc., 134:149-156, 2006.

[34] Yong Zhou and Sadek Gala. Logarithmically improved regularity criteria for the Navier-Stokes equations in multiplier spaces. Journal of Mathematical Analysis and Applications, 356(2):498-501, August 2009.

[35] Xiang'ou Zhu. A regularity criterion for the Navier-Stokes equations in the multiplier spaces. Abstract and Applied Analysis, 2012, 2012. 